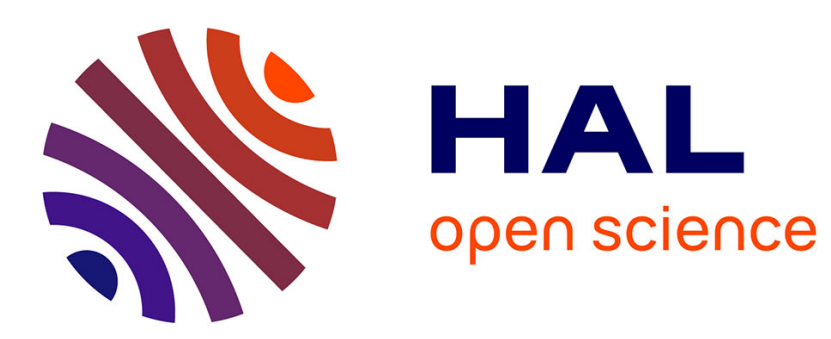

\title{
Electrowetting control of bouncing jets
}

Xavier Noblin, Franck Celestini

\section{To cite this version:}

Xavier Noblin, Franck Celestini. Electrowetting control of bouncing jets. Applied Physics Letters, 2012, 101, pp.094101. 10.1063/1.4747199 . hal-00727107

\section{HAL Id: hal-00727107 \\ https://hal.science/hal-00727107}

Submitted on 1 Sep 2012

HAL is a multi-disciplinary open access archive for the deposit and dissemination of scientific research documents, whether they are published or not. The documents may come from teaching and research institutions in France or abroad, or from public or private research centers.
L'archive ouverte pluridisciplinaire HAL, est destinée au dépôt et à la diffusion de documents scientifiques de niveau recherche, publiés ou non, émanant des établissements d'enseignement et de recherche français ou étrangers, des laboratoires publics ou privés. 


\title{
Electrowetting control of bouncing jets
}

\author{
Xavier Noblin a) and Franck Celestini ${ }^{\text {b) }}$ \\ Laboratoire de Physique de la Matière Condensée, UMR 6622, CNRS, Université de Nice Sophia-Antipolis, \\ Parc Valrose, 06108 Nice Cedex 2, France
}

(Received 29 February 2012; accepted 6 August 2012; published online 27 August 2012)

\begin{abstract}
As recently studied, a sub-millimetric liquid jet can bounce on sufficiently hydrophobic surfaces [F.Celestini et al., Soft Matter 6(23), $5872-5876$ (2010); A. Kibar et al., Exp. Fluids 49(5), $1135-1145$ (2010)]. As the hydrophobicity is reduced, the reflection angle $\left(\theta_{r}\right)$ increases and the jet rebound deviates more and more from specular reflection. In the present study, we vary the wetting properties of the substrate using the electrowetting effect to induce a change in the reflection angle. A liquid jet is sent toward a metallic surface coated by an insulating, hydrophobic layer. Applying an ac voltage between the metallic nozzle and the electrode below the insulating layer, we can precisely control the reflection angle of the jet. The effects of the amplitude and the frequency of the applied voltage are analyzed. This study can find applications for the control of jet dynamics. (C) 2012 American Institute of Physics. [http://dx.doi.org/10.1063/1.4747199]
\end{abstract}

A liquid jet impingement on a solid surface creates usually an hydraulic jump: $:^{3,4}$ a thin fluid layer after the impact zone followed by a transition to a thicker one. If the jet arrives normally on the surface, the jump is circular and the fluid remains on the substrate. When the jet incidence is non-normal on regular surfaces, the jump turns to an elongated shape but still remains stuck on the substrate. ${ }^{5}$ This behavior, which is also assumed in classical textbooks when dealing with such problem of jet impact, seems to follow an intuition since it corresponds to the common experience. Nevertheless, this situation is completely different when a photon or a solid ball impacts a flat surface: They bounce with a reflected angle $\left(\theta_{r}\right)$ equals to the incident one (Snell Descartes or specular reflection law). Drops can also bounce off a solid surface, ${ }^{6}$ the elasticity given by surface tension helps the drop to spring back and take off from the substrate. In the case of sub-millimetric jets, as recently studied, a bouncing can also be observed from sufficiently hydrophobic surfaces. The jet's bouncing is essentially governed by the Weber number $\left(W e=\left(\rho R U^{2}\right) /(\gamma)\right.$ with $U$ the jet velocity and $R$ the jet radius) that quantifies the relative importance of inertial versus capillary effects. ${ }^{7}$ This unexpected bouncing has been put in evidence in Refs. 2 and 1 for, respectively, large and small Weber numbers. We have explained in this later study that the normal component of the surface tension force can decrease the momentum transfer in the vertical direction, sticking the liquid jet to the substrate.

The electrowetting (EW) effect offers a way to continuously vary the wetting angle of a liquid drop deposited on a substrate. It has given rise to numerous applications, such as tunable focusing lens and droplets displacement control (for a review on this topic, see Refs. 8 and 9). Until now, this effect has been essentially used for drops although very recently Duez et al. ${ }^{10}$ applied it to control liquid flows out of a container.

In this letter, we show that the EW can be used to modify the direction of a bouncing jet. Taking advantage of the

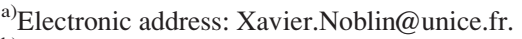

b) Electronic address: Franck.Celestini@unice.fr.
}

EW effect, we have continuously varied the wetting force and observed the change in the reflection angle $\left(\theta_{r}\right)$ of the jet. As expected, we confirm that when decreasing the wetting angle, the reflection angle $\theta_{r}$ increases up to $90^{\circ}$. This limiting case corresponds to a jet stuck on the substrate. We first describe the experimental setup and then present our measurements of $\theta_{r}$ as function of the applied voltage. We then focus on the effect of the frequency and finally discuss our results. To summarize, we demonstrate that the electrowetting effect, commonly applied to drops, can also be efficiently used for controlling the micro-jet dynamics.

The experimental set-up for the fluidic part of this experiment has been already described in detail. ${ }^{1}$ A slightly salted water jet is sent from a reservoir through a tube and a syringe nozzle towards the surface. This later is placed at $1 \mathrm{~cm}$ from the substrate. The jet velocity is controlled by the water pressure in the reservoir. We control the pressure value and a calibration curve permits to precisely measure the constant jet velocity. The incidence angle is noted $\theta_{i}$ (from the normal to the surface) and, as already mentioned, the reflection angle $\theta_{r}$. The jet radius is fixed to $195 \mu \mathrm{m}$, the jet velocity to $1.8 \mathrm{~m} / \mathrm{s}$ and $\theta_{i}$ to $50^{\circ}$. The salt concentration $(\mathrm{NaCl})$ is $2 \times 10^{-4} \mathrm{~mol} / \mathrm{l}$. The setup is presented in Fig. 1. The substrate is a flat piece of polished duraluminium coated with a $15 \mu \mathrm{m}$ layer of SU-8 2025 photoresist (Microchem) covered by a submicron layer of Teflon (AF 1600, Dupont). Both layers are electric insulators. The electrowetting conditions consist in applying an ac voltage between the metallic nozzle and the bottom of the substrate. The frequency ranges from $500 \mathrm{~Hz}$ to $3500 \mathrm{~Hz}$ and the rms value $\left(U_{\text {eff }}\right)$ between 0 and $150 \mathrm{~V}$. Salt has been added to increase the frequency response of the fluid to the electrowetting effect as discussed later. dc voltage has also been tried in our experiment, but as for classical EW control of drop wetting, it leads to charge accumulation. For ac voltage, this charge effect is most of the time suppressed. The electrowetting effect leads to a reduction of the contact angle with the applied voltage $U$, following the law: ${ }^{9} \cos (\theta)=\cos \theta_{E}+\frac{\epsilon_{0} \epsilon}{2 d \gamma} U^{2}$. As shown in Fig. 3, our system presents the classical parabolic behavior below $150 \mathrm{~V}$, before saturation. This characterizes the 


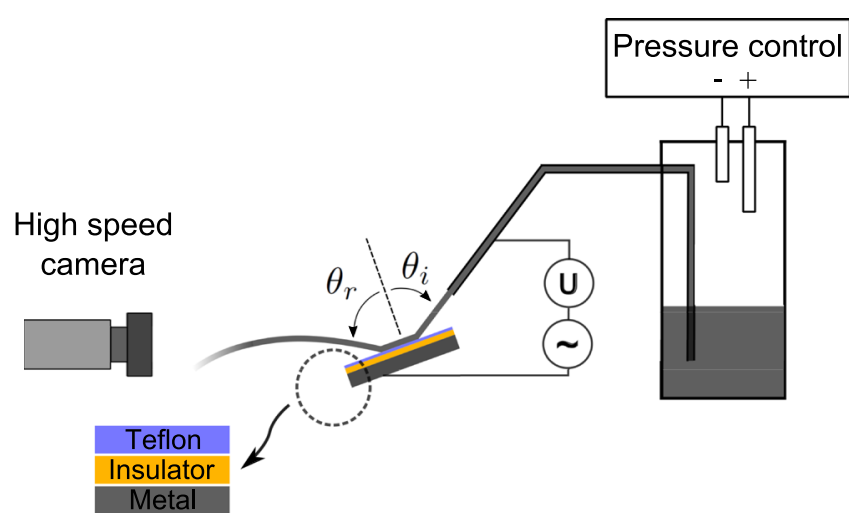

FIG. 1. Experimental setup showing the jet velocity control.

performance of our EW system for a drop deposited in quasistatic conditions.

The jet is continuously filmed by a high speed camera (Photron Ultima 512) at $250 \mathrm{fps}$ from the side. We then determined the jet trajectory after impact and deduced the reflection angle $\theta_{r}$ (see Fig. 2) from image analysis. Films at higher speed do not show any motion of the jet due to the ac frequency. This means that the mechanical response time of the jet was much higher than the ac period. The electrowetting effect is mainly linked to the average value of the square of the applied voltage. Filming at $250 \mathrm{fps}$ was then sufficient to measure the jet reflection angle. As stated later, long exposure time films also confirmed that no jet motion is induced by the high frequency of the ac voltage applied.
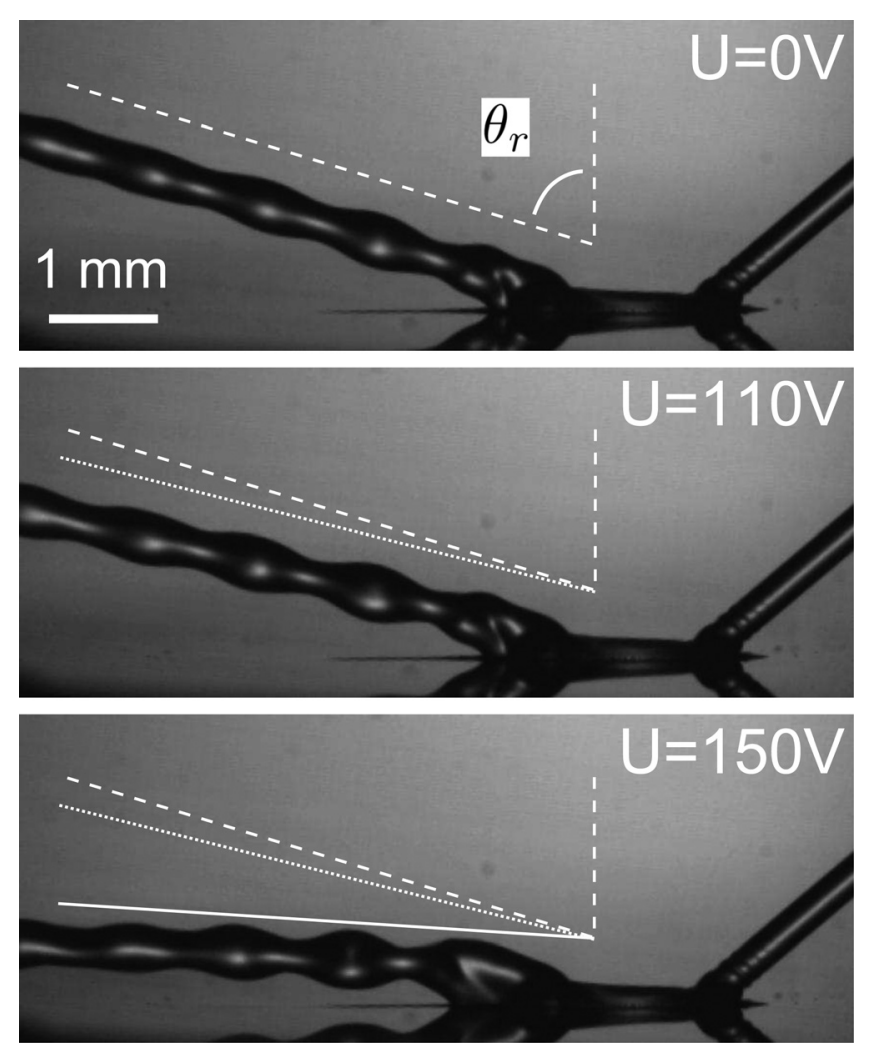

FIG. 2. Jet bouncing obtained for various applied voltages (from top to bottom: $0 \mathrm{~V}, 110 \mathrm{~V}$, and $150 \mathrm{~V}$ ). Lines are parallel to the jet after bouncing (dashed line: $0 \mathrm{~V}$, dotted line: $110 \mathrm{~V}$, and plain line: $150 \mathrm{~V}$ ).

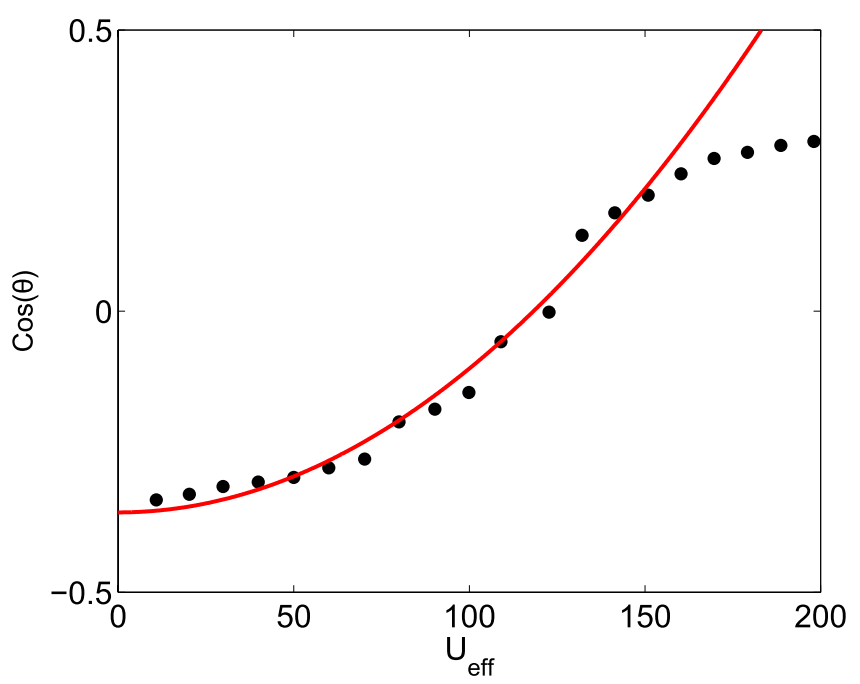

FIG. 3. Cosines of the wetting angle measured on a millimetric droplet versus rms voltage for an ac frequency of $500 \mathrm{~Hz}$. Plain line is the classical parabolic fit. The saturation phenomenon can be observed above $150 \mathrm{~V}$.

To evaluate the effect of the applied voltage, we have used a frequency $f=1 \mathrm{kHz}$ modulated in amplitude at a frequency $f_{m}=250 \mathrm{mHz}$. The applied voltage is therefore $U(t)=g(t) \sqrt{2} U_{\text {eff }} \cos (2 \pi f t)$. With $U_{\text {eff }}=150 \mathrm{~V}$ and $g(t) \mathrm{a}$ triangle function of frequency $f_{m}$ and values between 0 and 1. We take several images during $16 \mathrm{~s}$ corresponding to 4 periods of the modulated signal. We finally average the data and obtain the curve giving the reflection angle as a function of applied the voltage (Fig. 4). When the voltage is moderate, the jet remains at the same reflection angle. It appears to be very stable as confirmed by movies taken with a longer exposure without any blur effect. We can see that the effect on the reflection angle increases rapidly above $80 \mathrm{~V}$. Above $150 \mathrm{~V}$ the jet nearly touches the substrate. We have averaged the data distinguishing the points corresponding to an increase or a decrease of the applied voltage (respectively, blue circles and red squares in Fig. 4) without noticing any significant hysteric effect. When the jet touches the substrate, its motion is less stable and present fast oscillations that are out of the scope of the present letter.

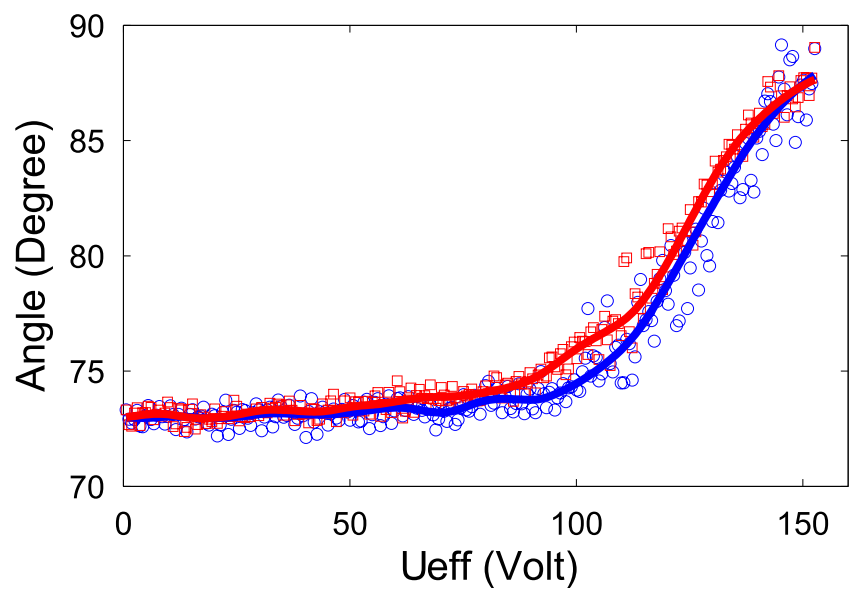

FIG. 4. Jet reflection angle as function of the ac voltage (rms value) for a constant frequency of $1 \mathrm{kHz}$. Blue circles: increasing rms voltage, red squares: decreasing $U_{\text {eff }}$. Plain curves are averaged data as a guide to the eyes. 


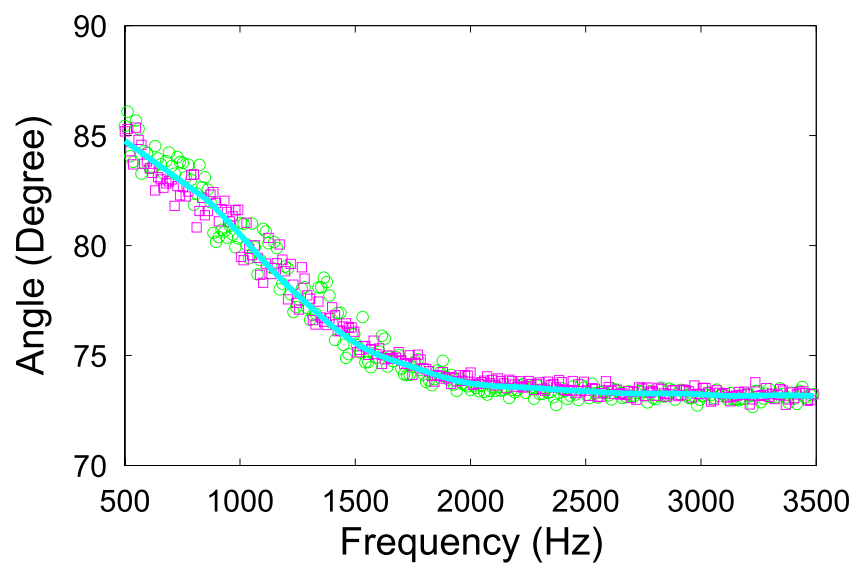

FIG. 5. Jet reflection angle as function of the ac frequency, for a constant rms voltage of $127 \mathrm{~V}$. Both colors are for increasing (green circles) and decreasing (magenta squares) frequencies. Plain curves are averaged data as a guide to the eyes.

For classical electro-wetting of drops, the contact angle cannot follow the high frequency of the ac applied voltage above a value that depends on the ionic concentration of the solution considered and the electrowetting geometry. When the frequency is increased above this limit value, the charges cannot follow the ac frequency and the electrowetting effect is suppressed. ${ }^{11-13} \mathrm{We}$ have therefore performed experiments to test the effect of the frequency on the variation of the reflection angle of bouncing jets. This has been realized in the same vein as for the voltage amplitude: A triangular shape modulation of the frequency is used and the applied voltage is $U(t)=\sqrt{2} U_{\text {eff }} \cos \left(2 \pi\left(\left(f_{\text {max }}-f_{\text {min }}\right) g(t)+f_{\text {min }}\right) t\right)$ with $g(t)$ the same triangle function of frequency $f_{m}=250 \mathrm{mHz}$, with value between 0 and 1 . We have used the amplitude $U_{\text {eff }}=127 \mathrm{~V}$, and a frequency varied from $f_{\text {min }}=500 \mathrm{~Hz}$ to $f_{\text {max }}=3500 \mathrm{~Hz}$. We plot the reflection angle versus the frequency in the Figure 5. One can see that the reflection angle is decreasing with the frequency. Above roughly $2 \mathrm{kHz}$ the electrowetting effect is almost negligible and the reflection angle almost unchanged compare to the situation without any applied voltage. As for the previous experiment, no hysteretic phenomenon has been observed. We demonstrate here that, for a sufficiently large applied voltage, the frequency can be used to precisely control the direction of the bouncing jet.

In the jet case with no electrowetting effect, as shown in Ref. 1, when the contact angle is decreased, the surface tension force increases and the reflection angle $\theta_{r}$ increases. Here, we confirm this effect by exploring a large range of contact angle values, leading to an increase in the reflected angle $\theta_{r}$ from $70^{\circ}$ to almost $90^{\circ}$. We can see that for low ac frequencies, the jet is sensitive to the applied voltage, and it is almost not reflected. As frequency is increased, the electrowetting effect disappears; the solution seems to behave as a dielectric and no longer as a perfect conductor. This effect is due to the critical frequency that limits the frequency range of electrowetting, ${ }^{11-13}$ it is given by $f_{c}=\frac{\sigma_{l} d / l}{\epsilon_{0}(\epsilon+81 d / l)} \cdot \epsilon_{d}$ is the relative permittivity of the insulator, $d=15 \mu \mathrm{m}$ is the insulator thickness, $l$ is the liquid thickness, and $\sigma_{l}=0.2 \mathrm{~S} / \mathrm{m}$ its conductivity. In our case, we have to take into account the geometry of the capacitor between the nozzle and the electrode below the insulator layer. Here, $l$ is the distance from the metallic nozzle to the substrate, its value is approximately $l=1 \mathrm{~cm}$. Applying the relationship for $f_{c}$ to our system leads to a limiting frequency of about $1.5 \mathrm{kHz}$. On Fig. 5, we clearly see that the critical frequency above which the electrowetting effect becomes null is around this value. We have a good agreement with the theory. A more quantitative comparison between geometry, flow conditions, and salt concentration will be addressed in a future study.

We have shown that electrowetting can be used to manipulate a liquid jet, and in particular to finely tune its direction after its impact on a hydrophobic surface. We confirm that reducing the substrate hydrophobicity prevents the jet from bouncing, this later remaining stuck on the surface. Both the frequency and the amplitude of the ac voltage applied are usable as a control parameter for jet microfluidics applications.

${ }^{1}$ F. Celestini, R. Kofman, X. Noblin, and M. Pellegrin, Soft Matter 6(23), 5872-5876 (2010).

${ }^{2}$ A. Kibar, H. Karabay, K. S. Yigit, I. O. Ucar, and H. Y. Erbil, Exp. Fluids 49(5), 1135-1145 (2010).

${ }^{3}$ C. Ellegaard, A. E. Hansen, A. Haaning, K. Hansen, A. Marcussen, T. Bohr, J. L. Hansen, and S. Watanabe, Nature 392, 767 (1998).

${ }^{4}$ J. Eggers and E. Villermaux, Rep. Prog. Phys. 71, 036601 (2008).

${ }^{5}$ R. P. Kate, P. K. Das, and S. Chakraborty, Phys. Rev. E 75, 056310 (2007).

${ }^{6}$ D. Richard and D. Quere, Europhys. Lett. 50(6), 769-775 (2000).

${ }^{7}$ C. Weber, Z. Angew. Math. Mech. 11(2), 136-154 (1931).

${ }^{8}$ B. Berge, C. R. Acad. Sci., Ser. II 317(2), 157-163 (1993).

${ }^{9}$ F. Mugele and J. C. Baret, J. Phys.: Condens. Matter 17(28), R705-R774 (2005).

${ }^{10}$ C. Duez, C. Ybert, C. Clanet, and L. Bocquet, Phys. Rev. Lett. 104(8), 084503 (2010).

${ }^{11}$ H. H. Woodson and J. R. Melcher, Electromechanical Dynamics (Wiley, New York, 1968), Vol. 2.

${ }^{12}$ D. A. Saville, Annu. Rev. Fluid Mech. 29, 27-64 (1997).

${ }^{13}$ F. Mugele, M. Duits, and D. van den Ende, Adv. Colloid Interface Sci. 161, 115-123 (2010). 